\title{
Silkworms with Spider Silklike Fibers Using Synthetic Silkworm Chow Containing Calcium Lignosulfonate, Carbon Nanotubes, and Graphene
}

Xiaoli Zhang, $^{\dagger}$ Ana Laura Licon, ${ }^{\ddagger}$ Thomas I. Harris, $^{\dagger}$ Paula F. Oliveira, ${ }^{\dagger}$ Bailey J. McFarland, ${ }^{\ddagger}$ Blake E. Taurone, ${ }^{\dagger}$ Brittney J. Walsh, ${ }^{\dagger}$ Brianne E. Bell, ${ }^{\dagger}$ Caleb T. Walker, ${ }^{\dagger}$ Randolph V. Lewis, ${ }^{\dagger, \dagger}$ and Justin A. Jones $* \dagger+(0)$

${ }^{\dagger}$ Departments of Biology and ${ }^{\ddagger}$ Departments of Biological Engineering, Utah State University, Logan, Utah 84322, United States

\section{Supporting Information}

ABSTRACT: Silkworm silk has become increasingly relevant for material applications. However, the industry as a whole is retracting because of problems with mass production. One of the key problems is the inconsistent properties of the silk. A means by which to improve the silk material properties is through enhanced sericulture techniques. One possible technique is altering the feed of the silkworms to include single-wall carbon nanotubes (SWNTs) or graphene (GR). Recently published results have demonstrated substantial improvement in fiber mechanical properties. However, the effect of the surfactant used to incorporate those materials into the feed on the fiber mechanical properties in comparison to normal silkworm silk has not been studied or reported.
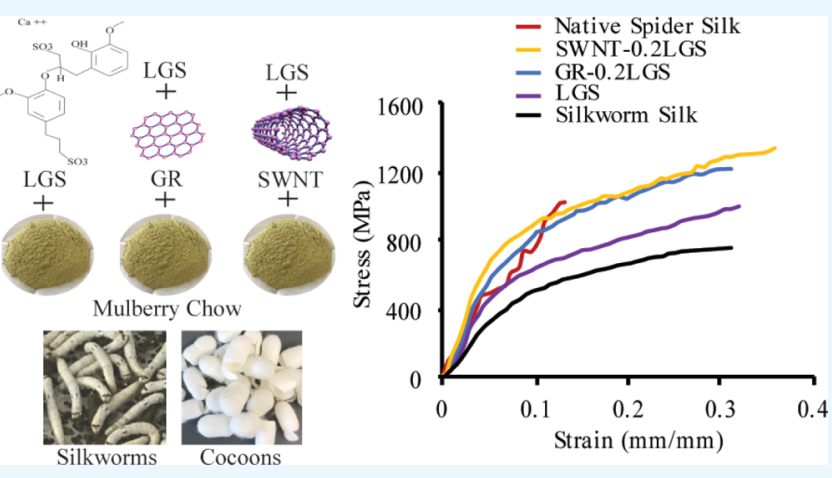
Thus, the total effect of feeding the SWNT and GR in the presence of surfactants on silkworms is not understood. Our study focuses on the surfactant [calcium lignosulfonate (LGS)] and demonstrates that it alone results in appreciable improvement of mechanical properties in comparison to nontreated silkworm silk. Furthermore, our study demonstrates that mixing the LGS, SWNT, and GR directly into the artificial diet of silkworms yields improved mechanical properties without decline below the control silk at high doses of SWNT or GR. Combined, we present evidence that mixing surfactants, in this case LGS, directly with the diet of silkworms creates a high-quality fiber product that can exceed $1 \mathrm{GPa}$ in tensile strength. With the addition of nanocarbons, either SWNT or GR, the improvement is even greater and consistently surpasses control fibers. However, feeding LGS alone is a more economical and practical choice to consistently improve the mechanical properties of silkworm fiber.

\section{INTRODUCTION}

Silkworm silk has been a highly utilized material, but its prevalence is declining for a variety of reasons. Primarily, the fragmented and varied nature of producing silkworm silk results in a wide variation of mechanical properties. ${ }^{1-4}$ The demand for high-performance silkworm fibers has led to efforts to improve sericulture techniques which, in turn, can improve fiber mechanical properties and consistency. ${ }^{2,5,6}$ Investigations have studied altered diets to affect fiber color and other physical fiber properties, which have clearly demonstrated that silkworms can incorporate compounds introduced during feeding directly into their fibers. ${ }^{7-14}$ More recent modifications have utilized the incorporation of single-wall carbon nanotubes (SWNTs) or graphene (GR) into the silk to improve the mechanical properties of the silkworm silk. ${ }^{15,16}$ These reported results have been remarkable. However, the studies do not completely elucidate the causative agents for the observed improvement, and the role of the surfactant, calcium lignosulfonate (LGS), used to disperse the SWNT and GR is not understood. ${ }^{15,17}$
Our research clarifies the effect of feeding LGS, SWNT with LGS, and GR with LGS to silkworms, and how these additives alter the protein structures and resultant final mechanical properties of the fibers. LGS is an abundant anionic detergent that is nontoxic. In previous publications, an important control was omitted to determine whether improved fiber mechanical properties is due, in part or completely, to the surfactant as opposed to the SWNT and GR. ${ }^{15}$ The mechanical properties of the reported control silk (normal silkworms fed only mulberry leaves sprayed with LGS) underperformed to what is typically observed for normal silkworm silk. ${ }^{1,15,18-20}$ Thus, the effect of the surfactant in the feed of the control silk (according to the authors, a normal silk control was not run) could impact the effect that feeding SWNT and GR plus surfactant on the materials properties of the silk fibers. Specifically, this research aims to discover the role of the surfactant alone as it relates to

Received: December 19, 2018

Accepted: February 20, 2019

Published: March 5, 2019 
Table 1. Summary of Mechanical Properties for All Fiber Groups and Respective Percent Changes

\begin{tabular}{|c|c|c|c|c|c|c|c|c|}
\hline & \multicolumn{4}{|c|}{ (A) maximum stress $(\mathrm{MPa})$} & \multicolumn{4}{|c|}{ (B) maximum strain $(\mathrm{mm} / \mathrm{mm})$} \\
\hline & mean & Std. Dev. & $\%$ change from control $^{a}$ & $\%$ change from LGS ${ }^{b}$ & mean & Std. Dev. & $\%$ change from control ${ }^{a}$ & $\%$ change from LGS $^{b}$ \\
\hline control & 751.7 & 110.6 & & & 0.31 & 0.04 & & \\
\hline LGS & 1014.1 & 176.5 & 34.9 & & 0.32 & 0.10 & 3.2 & \\
\hline GR-0.2LGS & 1211.2 & 322.3 & 61.1 & 19.4 & 0.31 & 0.09 & 0.0 & -3.1 \\
\hline GR-1LGS & 947.2 & 73.5 & 26.0 & -6.6 & 0.27 & 0.01 & -12.9 & -15.6 \\
\hline SWNT-0.2LGS & 1345.5 & 274.0 & 77.0 & 32.7 & 0.36 & 0.05 & 16.1 & 12.5 \\
\hline \multirow[t]{3}{*}{ SWNT-1LGS } & 1096.0 & 124.8 & 45.8 & 8.1 & 0.34 & 0.15 & 9.7 & 6.2 \\
\hline & \multicolumn{4}{|c|}{ (C) toughness $\left(\mathrm{MJ} / \mathrm{m}^{3}\right)$} & \multicolumn{4}{|c|}{ (D) elastic modulus (GPa) } \\
\hline & mean & Std. Dev. & $\%$ change from control $^{a}$ & $\%$ change from $\mathrm{LGS}^{b}$ & mean & Std. Dev. & $\%$ change from control ${ }^{a}$ & $\%$ change from LGS $^{b}$ \\
\hline control & 163.0 & 28.3 & & & 6.2 & 1.45 & & \\
\hline LGS & 218.6 & 68.1 & 34.1 & & 11.3 & 2.0 & 81.5 & \\
\hline GR-0.2LGS & 266.5 & 96.3 & 63.5 & 21.9 & 9.7 & 2.6 & 56.8 & 26.0 \\
\hline GR-1LGS & 180.6 & 13.5 & 10.8 & -17.4 & 10.9 & 1.0 & 76.3 & -50.0 \\
\hline SWNT-0.2LGS & 333.8 & 65.0 & 104.8 & 52.7 & 13.4 & 3.9 & 116.8 & 93.1 \\
\hline SWNT-1LGS & 260.6 & 119.8 & 59.9 & 19.2 & 9.4 & 4.6 & 52.3 & 123.0 \\
\hline
\end{tabular}

$a_{\%}$ change is determined from the differences between the mechanical properties of the control fibers and the mechanical properties of the other groups. ${ }^{b} \%$ change is determined from the differences between the mechanical properties of the LGS-treated fibers and the mechanical properties of the other groups except the controls.
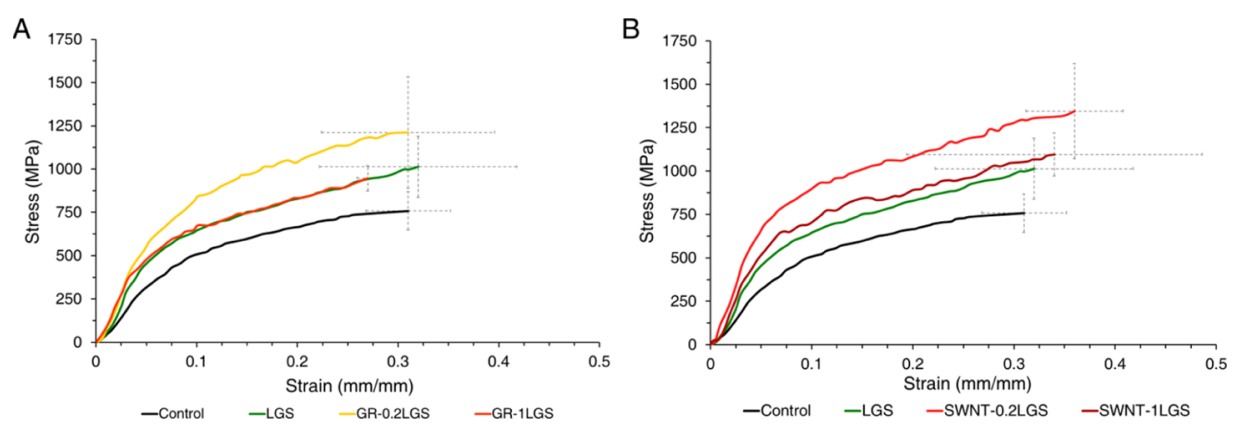

Figure 1. Representative stress-strain curves for the nanocarbon treatment groups. (A) Representative curves for control, LGS, GR-0.2LGS, and GR-1LGS fibers. (B) Representative curves for control, LGS, SWNT-0.2LGS, and SWNT-1LGS fibers.

fiber mechanical properties. Additionally, we seek to demonstrate an alternative, scalable approach to feeding LGS, SWNT, and GR to silkworms and ultimately validate the improvements seen in other studies through the addition of SWNT and GR while starting with high-performance silkworm silk.

\section{RESULTS}

Mechanical Properties. Compiled mechanical results are presented in Table 1, and representative stress-strain curves are presented in Figure 1. A control silkworm silk demonstrated average mechanical properties of $0.75 \mathrm{GPa}$ tensile strength, $0.31 \mathrm{~mm} / \mathrm{mm}$ strain, $6.2 \mathrm{GPa}$ elastic modulus, and $163 \mathrm{MJ} / \mathrm{m}^{3}$ toughness. Every treatment group saw an increase in average maximum tensile strength with the GR$0.2 \mathrm{LGS}$ and SWNT-0.2LGS treatment groups showing the greatest improvement of all (1.21 and $1.35 \mathrm{GPa}$, respectively). Average strain at fracture did not vary significantly between the treatment groups and the control group (Table 1, Figures 1 and S1).

Every treatment group saw an increased average elastic modulus when compared to the control $(6.2 \mathrm{GPa})$ group (Table 1D). The SWNT-0.2LGS treatment group showed the most improvement with an average elastic modulus of 13.44 $\mathrm{GPa}$, which was double the elastic modulus of the control group. The average toughness values (Table 1C) demonstrated increases for all treatment groups with the smallest increase in the GR-1LGS treatment group $\left(180.58 \mathrm{MJ} / \mathrm{m}^{3}\right)$. The highest performing group, for average energy-to-break, was the SWNT-0.2LGS treatment group which was more than doubled the energy-to-break of control silk from 163 to $333.76 \mathrm{MJ} / \mathrm{m}^{3}$. The LGS group saw an increase of $34.9 \%$ in tensile strength (Table 1A), only a small change in strain of $3.2 \%$ (Table 1B), a $34.1 \%$ increase in toughness (Table 1C), and an $81.5 \%$ increase in elastic modulus (Table 1D) compared to the control of normal silkworm silk. Of particular note is that the LGS group matched, or surpassed, the $1 \mathrm{GPa}$ threshold for tensile strength on average.

Overall, as shown in Table 1, increased values were produced for nearly all mechanical properties of the treatment groups when compared to the control silk, except for the maximum strains of both GR groups. Comparing the control and the best-performing treatment groups, the GR-0.2LGS had increases of 63.5 and $61.1 \%$ in toughness and tensile strength, respectively. The SWNT-0.2LGS had increases of 104.7 and $79.0 \%$ in toughness and tensile strength, respectively. However, when making the same comparison with GR, the GR-0.2LGS decreased by $1.2 \%$ in strain, whereas the SWNT-0.2LGS had an increase of $13.6 \%$.

When the mechanical properties are compared between groups that all contained LGS, using the LGS-only group as the new control, differences were still observed. The GR- 
0.2LGS has increases of only 21.9 and $19.4 \%$ in toughness and tensile strength, respectively. The SWNT-0.2LGS showed a $52.7 \%$ increase in toughness and an increase in tensile strength of $32.7 \%$. The GR-0.2LGS had a decrease in strain of $3.1 \%$, compared to the LGS-only group, whereas the SWNT$0.2 \mathrm{LGS}$ had an increase of $12.5 \%$.

Fourier Transform Infrared-Attenuated Total Reflectance Characterization. Structural characterization of the fibers from each treatment group revealed noticeable structural changes to the proteins that could potentially lead to the improved mechanical performance observed between the treatment groups and the control (Figure S2). Deconvolution of Fourier transform infrared (FTIR) spectra (Figures 2 and

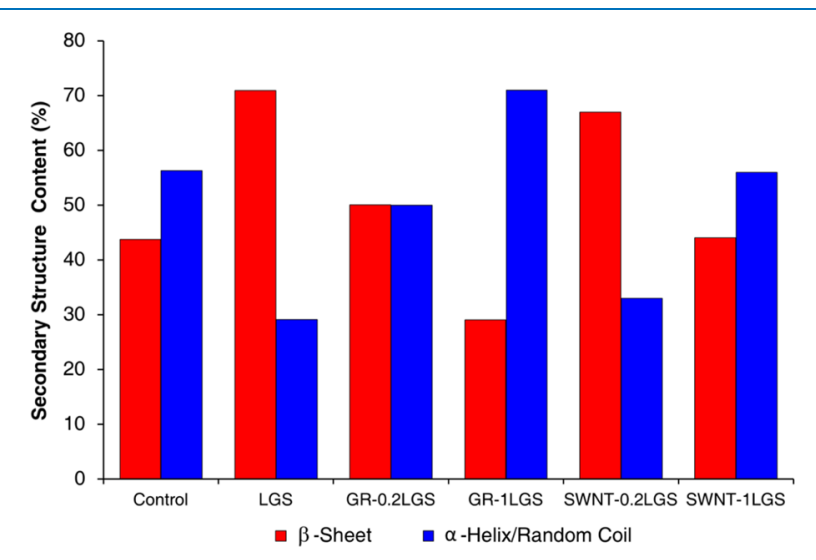

Figure 2. Protein secondary structure content of silk fibers. Varying $\beta$ sheet and $\alpha$-helix/random coil content of each fiber group as determined from analysis of the FTIR spectra.

S2) revealed that the control silk had a percentage of $\beta$-sheets at $44 \%$ and $\alpha$-helices/random coil at $56 \%$, similar to that reported in the literature. ${ }^{21-23}$ The addition of LGS to the diet alone increased the $\beta$-sheet content to $71 \%$. The GR-0.2LGS diet resulted in silk with $50 \% \beta$-sheet content, the GR-1LGS diet in fibers with $29 \% \beta$-sheet content, the SWNT-0.2LGS diet in fibers with $67 \% \beta$-sheet content, and the SWNT-1LGS in fibers with $44 \% \beta$-sheet content. The structural differences between the fibers of each group also suggest that the natural protein organization and structures are altered with the incorporation of the LGS, GR, or SWNT.
Microscopy Fiber Analysis. Scanning electron microscopy (SEM) revealed no notable morphological differences or features between fibers of the various treatment groups, as demonstrated in Figure 3. Standard optical bright-field observation also revealed no obvious morphological differences between treatment groups (Figure S3).

\section{DISCUSSION}

This study demonstrates that the consumption of LGS (anionic surfactant) by silkworms results in the appreciable improvement of the mechanical properties of the silk fibers (34.9\% increase in tensile strength, 3.2\% increase in strain, $34.1 \%$ increase in toughness, and $81.5 \%$ increase in elastic modulus). The increase is not as great as when SWNT or GR, at the $0.2 \mathrm{~g}$ level, is included with the LGS. However, given the very high cost of feeding either SWNT or GR in large-scale sericulture, the addition of LGS, and possibly other surfactants, represents a more practical means to improve fiber mechanical properties and presents an approach that warrants further exploration. The resulting LGS-only-treated silkworms produced silk fibers exceeding $1 \mathrm{GPa}$ on average for ultimate tensile strength when starting with the high-quality Haoyue strain of silkworms. Additionally, our results indicated that the LGS, SWNT, and GR can be mixed into the feed rather than the intensive spraying method previously reported with similar levels of improvement. ${ }^{15}$

This study further confirms that the mechanical properties of silkworm silk can also be substantially improved through the feeding of SWNT and GR. In a previous study, the best fibers produced from the incorporation of nanocarbons had strain increases of $10.0 \%(0.10 \mathrm{~mm} / \mathrm{mm})$ for GR and $34.1 \%(0.13$ $\mathrm{mm} / \mathrm{mm}$ ) for SWNT when compared to the controls of 0.94 $\mathrm{mm} / \mathrm{mm} .{ }^{15}$ The largest increases in the tensile strengths of the treated fibers from the controls, with an average value of 360 $\mathrm{MPa}$, were $58.3 \%$ (570 MPa) for GR and $63 \%$ (590 MPa) for SWNT. Finally, the energy-to-break values of the fibers were also improved $67.6 \%\left(38.04 \mathrm{MJ} / \mathrm{m}^{3}\right)$ for GR and $112.5 \%(\mathrm{MJ} /$ $\mathrm{m}^{3}$ ) for SWNT in comparison to the control silk of $22.7 \mathrm{MJ} /$ $\mathrm{m}^{3}$. These improvements brought the very best group to a tensile strength of $590 \mathrm{MPa}$, a strain of $0.13 \mathrm{~mm} / \mathrm{mm}$, and a toughness of $48.2 \mathrm{MJ} / \mathrm{m}^{3}$. By starting with a higher quality of silk, our methods produced silk fibers that, in our best groups, had an average tensile strength of $1345.5 \mathrm{MPa}$, a strain of 0.36
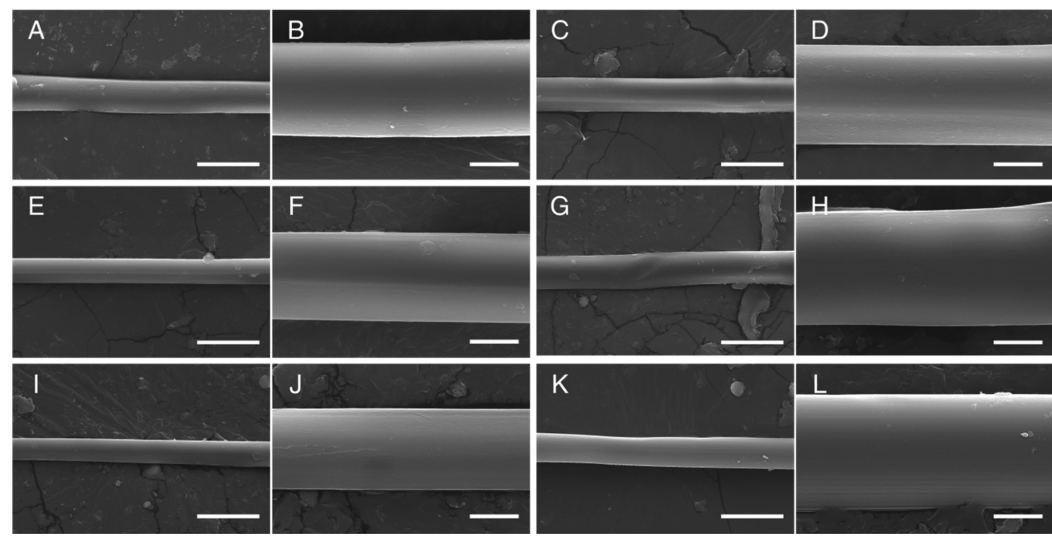

Figure 3. SEM micrographs of degummed silkworm fibers from each group at $2500 \times$ magnification (left) and $7500 \times$ magnification (right). (A,B) Control silkworm fibers. (C,D) LGS-treated silkworm fibers. (E,F) GR-0.2LGS-treated silkworm fibers. (G,H) GR-1LGS-treated silkworm fibers. (I,J) SWNT-0.2LGS-treated silkworm fibers. (K,L) SWNT-1LGS-treated silkworm fibers. Scale bars $=20$ and $5 \mu \mathrm{m}$ for $2500 \times$ and $7500 \times$, respectively. 
$\mathrm{mm} / \mathrm{mm}$, an elastic modulus of $13.44 \mathrm{GPa}$, and a toughness of $333.8 \mathrm{MJ} / \mathrm{m}^{3}$. These values approach or surpass the average mechanical properties of native spider silk fibers (Table S1). The groups with lower nanocarbon concentrations, SWNT$0.2 \mathrm{LGS}$ and GR-0.2LGS, exhibited the largest overall increases and highest final properties. Although the groups with higher nanocarbon concentrations (SWNT-1LGS and GR-1LGS) still surpassed the values of the control silk, the final properties were similar to those of that treated solely with LGS.

Although substantial increases in the final properties of the fibers occurred with the addition of SWNT and GR, this trend did not continue with the higher $1.0 \mathrm{~g}$ levels of nanocarbons. Overall, these fibers still had enhanced abilities when compared to the nontreated controls and were equivalent to the LGS-treated fibers, but more importantly the fibers with higher amounts (1 g) of SWNT and GR underperformed compared to fibers produced from lower nanocarbon amounts $(0.2 \mathrm{~g})$ (Figure 1 and Table 1$)$. This same negative effect, from increased nanocarbon additives, was also observed in the previously reported work, but with more drastic effects; the fibers produced from feeding higher amounts of SWNT and GR all suffered severely reduced properties that fell well under the control group (LGS for that study). ${ }^{15}$ Whereas the properties for the SWNT-1LGS and GR-1LGS in this study were similar to the LGS group and still above the control fibers. This feeding method may partially mitigate the negative effects of the higher amounts ( $1 \mathrm{~g})$ of SWNT and GR in the feed. This potentially prevents the silkworms from the overconsumption of SWNT or GR and thus, incorporating too much into their silk that can result in the nanocarbons acting as imperfections rather than improving fiber performance. As previously mentioned though, the benefits of simply introducing a surfactant into the feed seem a more practical approach for enhancing fiber properties, especially when higher concentrations of SWNT or GR are used.

The $\beta$-sheet content of the LGS treatment group alone was higher than the $\beta$-sheet content of the control group validating the increase of mechanical properties obtained with just the surfactant LGS. Interestingly, the greatest increase in $\beta$-sheet content was in the LGS-only group in comparison to the control. However, the mechanical properties of the LGS-only group were lower than both the GR-0.2LGS and SWNT$0.2 \mathrm{LGS}$ groups, even though the $\beta$-sheet content for these groups was lower than the LGS group. The $\beta$-sheet composition of the SWNT-1LGS and GR-1LGS groups was equivalent or lower than the control fibers and the $0.2 \mathrm{~g}$ nanocarbon treatments. Clearly, the content of $\beta$-sheets present within the fibers is not solely responsible for the mechanical properties of the silk; the arrangement of these structures within the fiber is also a factor. The lower $\beta$-sheet content of the $1 \mathrm{~g}$ treatments compared to the $0.2 \mathrm{~g}$ treatments indicates that the GR or SWNT at the higher doses is acting as imperfections disrupting the formation of $\beta$-sheets and their organization within the fiber. Even though the $0.2 \mathrm{~g}$ treatments have lower $\beta$-sheet content, the average mechanical properties exceed that of the LGS-alone group. The comparison of mechanical properties and structural characteristics indicates that the additions of GR and SWNT do indeed reinforce the fibers produced by the silkworms. However, the addition of SWNT and GR is not necessary to improve the abilities of native silkworm silk. Thus, LGS represents a scalable and economical alternative to enhance fiber mechanical properties through protein structure manipulation.

Neither bright-field microscopy nor SEM revealed changes to the appearance of the fibers. Degummed fibers from all of the groups appeared smooth without obvious imperfections or surface artifacts. This clearly suggests that the incorporation of LGS, SWNT, and GR into the feed does not have a detrimental effect on the traits or qualities of the fibers. However, at the $1 \mathrm{~g}$ dose of both GR and SWNT (GR-1LGS and SWNT-1LGS), the silkworms did not appear to be as healthy as the lower dosages of $0.2 \mathrm{~g}$ (GR-0.2LGS and SWNT-0.2LGS). Silkworms in the low dosage groups were slightly smaller in size and lethargic, whereas the high dosage groups appeared weaker and also had a higher mortality rate compared to the controls. Overall, there were no noticeable morphological differences in the final cocoons produced by the silkworms (Figure S4).

Clearly, the enrichment of silkworm feed with nanocarbons has a beneficial outcome on the final fibers. However, there are large differences between the final values reported in this study and those previously reported. This could partly be due to the higher performing silkworms and their fibers as the starting point for improvement. Additional factors that cannot be ruled out given the direct impact they can have on cocoons, and ultimately fiber properties are feeding methods, differences in sericulture conditions, silk harvesting and processing techniques, and testing parameters. ${ }^{2,12,15,24,25}$ All of these could potentially be future areas of investigation, with surfactant and nanocarbon additives, to further improve the consistency and properties of silkworm fibers. For example, testing speeds have an obvious influence on the mechanical properties. Generally, increasing the strain rate improves fiber and polymer mechanical characteristics. ${ }^{26-29}$ Furthermore, higher test speeds are more representative of practical rates of strain that materials are subjected to. As a means of comparison, the control silk in this study was tested at $1 \mathrm{~mm} / \mathrm{min}$ as was previously reported by Wang et al. The control silk tested at 1 $\mathrm{mm} / \mathrm{min}$ had a tensile strength of $502.7 \mathrm{MPa}$ but when tested at $500 \mathrm{~mm} / \mathrm{min}$ had a strength of $751.7 \mathrm{MPa}$.

In previous publications, the presence of GR and SWNT was confirmed in the silk of the silkworms and even the excrement of the silkworms. ${ }^{15,30}$ The surfactant is much more difficult to assess whether it is present within the fiber. Further work will confirm the presence of the surfactant. Our study does not elucidate the uptake mechanism, incorporation, or presence of the LGS into the fiber. It is possible that the surfactant could act to improve gut permeability and increase nutritional support to the silkworm thus improving fiber mechanical properties.

\section{CONCLUSIONS}

Our study demonstrated that the addition of LGS (an anionic surfactant) to the feed of silkworms improves the mechanical properties of the resulting fibers to near native spider silk values. The designation of the silkworm fibers as spider silk-like was supported and validated through the testing of native spider silk fibers, as reported in Table S1. This study also confirms that feeding low doses of nanocarbons with the surfactant ( $0.2 \mathrm{~g}$ of GR and SWNT), when starting with a high-quality silkworm strain, can substantially improve the mechanical performance of the fibers to native spider silk mechanical levels. ${ }^{20,31,32}$ Additionally, it has been demonstrated through this study that the GR and SWNT can be 
Table 2. Feed Specifications and Ratios for the Quantities of LGS, GR, and SWNT Prepared for Each Specific Experimental Group

\begin{tabular}{|c|c|c|c|c|}
\hline silkworm treatment group & chow quantity (g) & LGS/chow (g) & nanocarbon/chow (g) & group label \\
\hline control & & 0 & 0 & control \\
\hline LGS & 100 & 5 & 0 & LGS \\
\hline \multirow{2}{*}{ GR } & & & 0.2 & GR-0.2LGS \\
\hline & & & 1 & GR-1LGS \\
\hline \multirow[t]{2}{*}{ SWNT } & & & 0.2 & SWNT-0.2LGS \\
\hline & & & 1 & SWNT-1LGS \\
\hline
\end{tabular}

mixed into the feed with relative ease and precision to achieve marked improvement in fiber mechanical properties. This method appears to reduce the negative effect on the mechanical properties when feeding higher amounts of SWNT and GR than previously reported. ${ }^{15}$

\section{MATERIALS AND METHODS}

Feed Preparation and Feeding Procedures. A purebred strain of silkworms (Haoyue) with white cocoons was used for this study. A commercial silkworm powdered chow diet was obtained from Mulberry Farms (Fallbrook, CA, USA). The feedstock was prepared according to the manufacturer's directions. SWNTs, with an average diameter of $1.1 \mathrm{~nm}$ and length of 5-30 $\mu \mathrm{m}$ (US4110, US Research Nanomaterials, Houston, TX, USA), and Graphene, with average particle size $<50 \mathrm{~nm}$ and specific surface area of $30-50 \mathrm{~m}^{2} / \mathrm{g}$ (BST-Nano, San Diego, CA, USA), were added to the chow in the amounts listed in Table 2 and in the same ratios reported in a previous study. ${ }^{15}$ In order to assure complete and homogeneous mixing of the LGS, SWNT, and GR additives, they were added as dry powders to the dry silkworm chow and thoroughly mixed by hand. Water was then added, and the combinations were thoroughly mixed again. The wet chow was placed in a microwave for heating to prepare the final feedstock. Food was administered to the worms at a consistent level by weighing prior to feeding, thus ensuring that one group was not fed more than others. A conventional pasta maker was utilized to shape the feed uniformly into pasta-like ribbons to ensure even distribution of feed. Feed was not limited. Feeding SWNT and GR enriched silkworm chow commenced at the beginning of the third instar with each group of silkworms containing 10 individuals, similar to previously reported methods. ${ }^{15}$

The silkworm chow for each group was replaced every one to two days, as needed. Worms were allowed to develop to the end of the fifth instar at which point they were transferred to rolled paper tubes measuring $30 \mathrm{~mm}$ in diameter and $225 \mathrm{~mm}$ in length to spin their cocoons, with three worms in each tube. Both ends of the tubes were secured after placing the silkworms in it to prevent escape and to promote successful cocoon formation. At completion of cocoon spinning, the tubes were opened and cocoons retrieved.

Fiber Degumming. After cocoon production, the cocoons were cut open with a small blade at one end and the pupae were removed. Segments from each cocoon were gently removed by cutting. A degumming (sericin removal) solution was prepared with $0.25 \%(\mathrm{w} / \mathrm{v}) \mathrm{Na}_{2} \mathrm{CO}_{3}$ in water. Each cocoon thread segment was placed with $80 \mathrm{~mL}$ of the degumming solution in a $100 \mathrm{~mL}$ glass bottle. The bottles were placed in a water bath at $85{ }^{\circ} \mathrm{C}$ and mixed constantly until the sericin was dissolved, indicated by the silk becoming more transparent and separating into a cloud-like shape. The silk was collected from the bottles and rinsed in water before air drying at room temperature on a flat surface to form a silk mat. Individual fiber segments were taken from each mat without pulling or stretching the fibers to preserve mechanical properties for mechanical testing.

Preparation and Mechanical Testing of Fibers. At the completion of silk degumming, rinsing, and drying, individual fibers were mounted on plastic film "C" shaped cards across a precut opening with a gauge length of $19.05 \mathrm{~mm} .{ }^{33}$ Three cocoons from each group were used for mechanical testing with three fibers from each cocoon. The fibers were then secured with cyanoacrylate glue at both ends of the fiber to the "C" card. Fiber diameters were measured using the Motic Image Plus 2.0 program and a Motic BA310 microscope at nine individual points along the $19.05 \mathrm{~mm}$ length.

Reported diameters are considered an average diameter. Silkworm fibers are composed of two fibers; thus, their "diameter" varies depending on the angle that the fiber is viewed with one angle showing essentially the width of a single fiber and the other showing the width of two fibers. The fiber(s) are twisted as a result of production by the silkworm. It is difficult, if not impossible, to scan the fiber under high magnification and determine where the twists start and stop, making diameter measurements less precise. ${ }^{24}$ Therefore, the most efficient method to measure diameters is to simply scan the fiber for the thinnest sections ensuring a consistent measurement. There is no formalized or consistent means by which to efficiently and accurately overcome these basic obstacles in fiber cross-sectional measurements, and many of the silk mechanical properties reported in the literature have unclear, or completely omit, methods for determining the sample cross-sectional areas. ${ }^{3,15,30,34-37}$ However, all fibers reported in this study were measured and treated equally using the method described above.

Fibers were tested with environmental conditions of $22{ }^{\circ} \mathrm{C}$ and $40 \%$ humidity on a MTS Synergie 100 instrument, with a custom $10 \mathrm{~g}$ load cell, to measure the uniaxial tensile mechanical properties at $500 \mathrm{~mm} / \mathrm{min}$. Additionally, our control silk was also tested at $1 \mathrm{~mm} / \mathrm{min}$ for comparison to Wang et al. where test speeds were reported at $1 \mathrm{~mm} / \mathrm{min}$ (Table S2). The data gathered were then exported as a .txt file and imported into Microsoft Excel to further determine and calculate the mechanical properties of the fibers and the effects of the individual treatment groups.

SEM Morphological Analysis. Morphological analysis of the fibers was performed with a Quanta FEG 650 field emission scanning electron microscope. Three fiber samples from each treat group were randomly collected from the degummed fiber mats. All of the fibers were then stretched out and mounted on a carbon tape with all fibers parallel to each other. The prepared sample holders and the samples were then sputter coated with a $10 \mathrm{~nm}$ layer of $\mathrm{Au} / \mathrm{Pd}$. Imaging of the sample was performed in high vacuum mode at $1.22 \mathrm{mPa}$ and 
collected with an Everhart-Thornley detector. Images were taken at $2500 \times$ and $7500 \times$ magnifications with a beam energy of $20 \mathrm{kV}$, a spot size of 2 , and a dwell time of $15 \mu \mathrm{s}$.

Spectroscopic Structural Characterization. The protein structures of the silkworm fibers were probed with Raman and FTIR spectroscopy. A Varian 660-IR instrument with a horizontal attenuated total reflectance (ATR) attachment was used for the FTIR spectroscopy. Background scans were obtained prior to the sample measurements. Fiber mats from each treatment were placed directly on the ATR crystal and clamped, and measurements were performed using the data acquisition software Resolution Pro Version. Each mat was measured multiple times with each measurement having the following parameters: an aperture setting of $4 \mathrm{~cm}^{-1}$ at 4000 $\mathrm{cm}^{-1}$, a resolution of $1 \mathrm{~cm}^{-1}$, and 32 scans over the range of $600-4000 \mathrm{~cm}^{-1}$. Deconvolution of the obtained FTIR spectra was performed with Origin data analysis software using a method previously reported by $\mathrm{Hu}$ et al. ${ }^{38}$ Minor differences in the method consisted of the use of a Gaussian fit and Origin instead of Opus 5.0. In brief, a nine-point Savitsky-Golay smoothing filter was applied with a 0.3 ratio; the fraction of the interferogram to which the apodization and deconvolution were applied. The assignments of vibrational bands previously used by Guo et al. were utilized for this analysis. ${ }^{39}$ The Gaussian line profiles were fit through a three-step process as follows: fixing the number and position of bands, allowing the band positions to shift in accordance with the LevenbergMarquardt algorithm, and finally adjusting the overall fitted curve with a nonlinear least-squared method to obtain a fit as near to the original spectra as possible. The areas of the resultant bands were analyzed to determine the secondary protein structure contents within the fibers for the amide I region.

A Renishaw inVia Raman microscope with a $633 \mathrm{~nm} \mathrm{HeNe}$ laser was used for the Raman spectroscopy. Bundles of fibers were stretched across an aluminum tape on a glass slide and then secured at both ends. Spectral acquisitions were obtained with RenishawWire 4.1 after the bundles were focused with the $20 \times$ objective. Each bundle was measured multiple times with each acquisition having the following parameters: an exposure time of $60 \mathrm{~s}$ at $100 \%$ laser power, a range from 200 to 3200 $\mathrm{cm}^{-1}$, with a total of 5 accumulations. Finally, after completing the spectral acquisition, the background was subtracted for each measurement.

\section{ASSOCIATED CONTENT}

\section{S Supporting Information}

The Supporting Information is available free of charge on the ACS Publications website at DOI: 10.1021/acsomega.8b03566.

Average mechanical properties chart for all silkworm treatment groups; spectroscopy spectra of all silkworm treatment groups; bright-field microscopy images of fibers; mechanical properties of natural dragline silk from Nephila clavipes; cocoon morphology; and mechanical properties of control fibers at different test speeds (PDF)

\section{AUTHOR INFORMATION}

\section{Corresponding Author}

*E-mail: justin.a.jones@usu.edu.

\section{ORCID}

Justin A. Jones: 0000-0002-3647-5361

\section{Notes}

The authors declare no competing financial interest.

\section{ACKNOWLEDGMENTS}

The authors would like to thank the Utah Science Technology and Research (USTAR) initiative for their financial support of this work. We would also like to thank Dr. Elizabeth Vargis for use of the Raman instrument. Finally, we acknowledge the support from the Microscopy Core Facility at Utah State University for the SEM results.

\section{ABBREVIATIONS}

GR, graphene; SWNT, single-wall carbon nanotube; LGS, calcium lignosulfonate.

\section{REFERENCES}

(1) Vepari, C.; Kaplan, D. L. Silk as a Biomaterial. Prog. Polym. Sci. 2007, 32, 991-1007.

(2) Rahmathulla, V. K.; Suresh, H. M. Management of Climatic Factors for Successful Silkworm (Bombyx mori) Crop and Higher Silk Production: A Review. Psyche 2012, 12, 1-14.

(3) Pérez-Rigueiro, J.; Viney, C.; Llorca, J.; Elices, M. Mechanical Properties of Single-Brin Silkworm Silk. J. Appl. Polym. Sci. 2000, 75, $1270-1277$.

(4) Kumaresan, P. Quality Silk Production: Some Economic Issues. Econ. Polit. Wkly. 2002, 37, 4019-4022.

(5) Nagaraju, J. Application of Genetic Principles for Improving Silk Production. Curr. Sci. 2002, 83, 409-414.

(6) Jiang, L.; Xia, Q. The Progress and Future of Enhancing Antiviral Capacity by Transgenic Technology in the Silkworm Bombyx mori. Insect Biochem. Mol. Biol. 2014, 48, 1-7.

(7) Sarker, A. A.; Haque, M. R.; Rab, M. A.; Absar, N. Effects of Feeding Mulberry (Morus Sp.) Leaves Supplemented with Different Nutrients to Silkworm (Bombyx mori). Curr. Sci. 1995, 69, 185-188.

(8) Wang, X.; Zhao, P.; Li, Y.; Yi, Q.; Ma, S.; Xie, K.; Chen, H.; Xia, Q. Modifying the Mechanical Properties of Silk Fiber by Genetically Disrupting the Ionic Environment for Silk Formation. Biomacromolecules 2015, 16, 3119-3125.

(9) Wang, J.-T.; Li, L.-L.; Feng, L.; Li, J.-F.; Jiang, L.-H.; Shen, Q. Directly Obtaining Pristine Magnetic Silk Fibers from Silkworm. Int. J. Biol. Macromol. 2014, 63, 205-209.

(10) Nicodemo, D.; Oliveira, J. E.; Sedano, A. A.; Marconcini, J. M.; Tonoli, G. H. D. Impact of Different Silkworm Dietary Supplements on Its Silk Performance. J. Mater. Sci. 2014, 49, 6302-6310.

(11) Iizuka, T.; Sezutsu, H.; Tatematsu, K.-i.; Kobayashi, I.; Yonemura, N.; Uchino, K.; Nakajima, K.; Kojima, K.; Takabayashi, C.; Machii, H.; et al. Colored Fluorescent Silk Made by Transgenic Silkworms. Adv. Funct. Mater. 2013, 23, 5232-5239.

(12) Tansil, N. C.; Koh, L. D.; Han, M.-Y. Functional Silk: Colored and Luminescent. Adv. Mater. 2012, 24, 1388-1397.

(13) Nisal, A.; Trivedy, K.; Mohammad, H.; Panneri, S.; Sen Gupta, S.; Lele, A.; Manchala, R.; Kumar, N. S.; Gadgil, M.; Khandelwal, H.; et al. Uptake of Azo Dyes into Silk Glands for Production of Colored Silk Cocoons Using a Green Feeding Approach. ACS Sustainable Chem. Eng. 2014, 2, 312-317.

(14) Tansil, N. C.; Li, Y.; Teng, C. P.; Zhang, S.; Win, K. Y.; Chen, X.; Liu, X. Y.; Han, M.-Y. Intrinsically Colored and Luminescent Silk. Adv. Mater. 2011, 23, 1463-1466.

(15) Wang, Q.; Wang, C.; Zhang, M.; Jian, M.; Zhang, Y. Feeding Single-Walled Carbon Nanotubes or Graphene to Silkworms for Reinforced Silk Fibers. Nano Lett. 2016, 16, 6695-6700.

(16) Ling, S.; Li, C.; Adamcik, J.; Wang, S.; Shao, Z.; Chen, X.; Mezzenga, R. Directed Growth of Silk Nanofibrils on Graphene and Their Hybrid Nanocomposites. ACS Macro Lett. 2014, 3, 146-152. 
(17) Ayutsede, J.; Gandhi, M.; Sukigara, S.; Ye, H.; Hsu, C.-M.; Gogotsi, Y.; Ko, F. Carbon Nanotube Reinforced Bombyx mori Silk Nanofibers by the Electrospinning Process. Biomacromolecules 2006, 7, 208-214.

(18) Cunniff, P. M.; Fossey, S. A.; Auerbach, M. A.; Song, J. W.; Kaplan, D. L.; Adams, W. W.; Eby, R. K.; Mahoney, D.; Vezie, D. L. Mechanical and Thermal Properties of Dragline Silk from the Spider Nephila clavipes. Polym. Adv. Technol. 1994, 5, 401-410.

(19) Shao, Z.; Vollrath, F. Materials: Surprising Strength of Silkworm Silk. Nature 2002, 418, 741.

(20) Gosline, JM; Guerette, PA; Ortlepp, CS; Savage, KN The Mechanical Design of Spider Silks: From Fibroin Sequence to Mechanical Function. J. Exp. Biol. 1999, 202, 3295-303.

(21) Lefêvre, T.; Rousseau, M.-E.; Pézolet, M. Protein Secondary Structure and Orientation in Silk as Revealed by Raman Spectromicroscopy. Biophys. J. 2007, 92, 2885-2895.

(22) Drnovšek, N.; Kocen, R.; Gantar, A.; Drobnič-Košorok, M.; Leonardi, A.; Križaj, I.; Rečnik, A.; Novak, S. Size of Silk Fibroin $\beta$ Sheet Domains Affected by $\mathrm{Ca}^{2+}$. J. Mater. Chem. B 2016, 4, 65976608.

(23) Asakura, T.; Okushita, K.; Williamson, M. P. Analysis of the Structure of Bombyx mori Silk Fibroin by NMR. Macromolecules 2015, 48, 2345-2357.

(24) Pérez-Rigueiro, J.; Viney, C.; Llorca, J.; Elices, M. Silkworm Silk as an Engineering Material. J. Appl. Polym. Sci. 1998, 70, 2439-2447.

(25) Jiang, P.; Liu, H.; Wang, C.; Wu, L.; Huang, J.; Guo, C. Tensile Behavior and Morphology of Differently Degummed Silkworm (Bombyx mori) Cocoon Silk Fibres. Mater. Lett. 2006, 60, 919-925.

(26) Swallowe, G. M. Strain Rate Effects. In Mechanical Properties and Testing of Polymers: An A-Z Reference; Swallowe, G. M., Ed.; Polymer Science and Technology Series; Springer Netherlands: Dordrecht, 1999; pp 214-218.

(27) Siviour, C. R.; Jordan, J. L. High Strain Rate Mechanics of Polymers: A Review. J. Dyn. Behav. Mater. 2016, 2, 15-32.

(28) Carmichael, S.; Viney, C. Molecular Order in Spider Major Ampullate Silk (Dragline): Effects of Spinning Rate and Post-Spin Drawing. J. Appl. Polym. Sci. 1999, 72, 895-903.

(29) Shim, V. P. W.; Lim, C. T.; Foo, K. J. Dynamic Mechanical Properties of Fabric Armour. Int. J. Impact Eng. 2001, 25, 1-15.

(30) Wang, J.-T.; Li, L.-L.; Zhang, M.-Y.; Liu, S.-L.; Jiang, L.-H.; Shen, Q. Directly Obtaining High Strength Silk Fiber from Silkworm by Feeding Carbon Nanotubes. Mater. Sci. Eng., C 2014, 34, 417-421.

(31) Swanson, B. O.; Blackledge, T. A.; Beltrán, J.; Hayashi, C. Y. Variation in the Material Properties of Spider Dragline Silk across Species. Appl. Phys. A 2005, 82, 213-218.

(32) Blackledge, T. A.; Hayashi, C. Y. Silken Toolkits: Biomechanics of Silk Fibers Spun by the Orb Web Spider Argiope argentata (Fabricius 1775). J. Exp. Biol. 2006, 209, 2452-2461.

(33) Stauffer, S. L.; Coguill, S. L.; Lewis, R. V. Comparison of Physical Properties of Three Silks from Nephila clavipes and Araneus gemmoides. J. Arachnol. 1994, 22, 5-11.

(34) Zhao, H.-P.; Feng, X.-Q.; Yu, S.-W.; Cui, W.-Z.; Zou, F.-Z. Mechanical Properties of Silkworm Cocoons. Polymer 2005, 46, 9192-9201.

(35) Lepore, E.; Bosia, F.; Bonaccorso, F.; Bruna, M.; Taioli, S.; Garberoglio, G.; Ferrari, A. C.; Pugno, N. M. Spider Silk Reinforced by Graphene or Carbon Nanotubes. 2D Mater. 2017, 4, 031013.

(36) Cheung, H.-Y.; Lau, K.-T.; Tao, X.-M.; Hui, D. A Potential Material for Tissue Engineering: Silkworm Silk/PLA Biocomposite. Composites, Part B 2008, 39, 1026-1033.

(37) Zhang, K.; Si, F. W.; Duan, H. L.; Wang, J. Microstructures and Mechanical Properties of Silks of Silkworm and Honeybee. Acta Biomater. 2010, 6, 2165-2171.

(38) Hu, X.; Kaplan, D.; Cebe, P. Determining Beta-Sheet Crystallinity in Fibrous Proteins by Thermal Analysis and Infrared Spectroscopy. Macromolecules 2006, 39, 6161-6170.

(39) Guo, C.; Zhang, J.; Jordan, J. S.; Wang, X.; Henning, R. W.; Yarger, J. L. Structural Comparison of Various Silkworm Silks: An
Insight into the Structure-Property Relationship. Biomacromolecules 2018, 19, 906-917. 